\title{
New occurrences of aphyllophoroid fungi (Agaricomycetes, Basidiomycota) in the Central Forest State Biosphere Nature Reserve, Tver Region, Russia
}

\author{
Ilya A. Viner ${ }^{1}$, Dmitry S. Schigel ${ }^{2} \&$ Heikki Kotiranta $^{3}$ \\ ${ }^{1}$ Faculty of Biology, Leninskie Gory 1-12, 119234, Lomonosov Moscow State University, Russia. \\ E-mail: viner.ilya@gmail.com \\ ${ }^{2}$ Departmen of Biosciences, P.O. Box 65, FI-00014, University of Helsinki, Finland. \\ E-mail: dmitry.shchigel@helsinki.fi \\ ${ }^{3}$ Finnish Environment Institute, Biodiversity Unit, P.O. Box 140, FI-00251 Helsinki, Finland. \\ E-mail: heikki.kotiranta@ymparisto.fi
}

\begin{abstract}
New data on non-agaricoid wood-inhabiting basidiomycetes collected from dead wood in the Central Forest Nature Reserve are provided. In total, 228 species were recorded during short-term visits in 2009-2015, of which 37 are reported as new to the reserve. An annotated species list is presented including details of associated substrata and, when available, personal fungarium specimen numbers. The paper increases the total number of species reported for the reserve and provides notes on specimens belonging to the genus Phlebiella, which is probably an undescribed species. Records of some rare, or rarely collected species, such as Antrodiella foliaceodentata, Basidiodendron radians, Phlebiella fibrillosa and Tulasnella eichleriana are discussed.
\end{abstract}

Keywords: Polypores, corticioid, biodiversity, dead wood

\section{INTRODUCTION}

The first study focusing on the biodiversity of aphyllophoroid fungi in the Central Forest Nature Reserve was carried out by V. A. Chastukhin and published in the manuscript "Mykoflora of the Central Forest Reserve, 1942". This is now unfortunately lost and known only from references in Chastukhin (1948) and Trofimov (1948). Moreover, it is not clear what happened to the specimens collected by Chastukhin: only a limited number of his collections are deposited in the Mycological fungarium of the Komarov Botanical Institute of RAS, St. Petersburg (Kotkova, 2014b). Nevertheless some data on the fungal biodiversity of the reserve can be found in Chastukhin (1948) and Chastukhin \& Nikolaevskaya (1969). Between 1975-1976 M. A. Bondartseva visited the reserve and provided a list of 107 species of wood-inhabiting basidiomycetes (Bondartseva, 1986). V. G. Storozhenko contributed to the data on biodiversity of the reserve by studying the ecology of 38 pathogenic wood-decaying fungi (Storozhenko, 2007) and preliminary reports on aphyllophoroid fungi were also made by Viner (2012). Between 2011-2012, V. M. Kotkova collected aphyllophoroid fungi in the northern and southern parts of the reserve (Kotkova, 2012a-c, 2014a) and summarized all the available data on its fungal biodiversity (Kotkova, 2014b).

The reserve is located in the southern part of the Valdai Hills where the southern taiga zone enters the mixed coniferous-broadleaved forests (Karpov, 1983, www.clgz.ru). This geographical location determines the high diversity of the host species available for wood-inhabiting fungi. In addition, the study area near Fyodorovskoe harbours a mix of vast forest areas unmanaged for hundreds of years before exploitation began in the 1950s (Pugachevsky \& Shaposnikhov, 1999). All these factors provide a variety of suitable environmental conditions for wood-inhabiting fungi and high species diversity. We report the extended list of species from the reserve and discuss and illustrate a corticioid species which is probably new to science.

\section{MATERIALS AND METHODS}

Fruit body inventories were made in the Central Forest State Biosphere Reserve in Tver Region, central European Russia (Fig 1), near Fyodorovskoe village $\left(56^{\circ} 26^{\prime}-31^{\prime} \mathrm{N}, 32^{\circ} 29^{\prime}-33^{\circ} 29^{\prime} \mathrm{E}\right)$. The field work was carried out from July to September in the years 2009-2015 at seven forest sites 


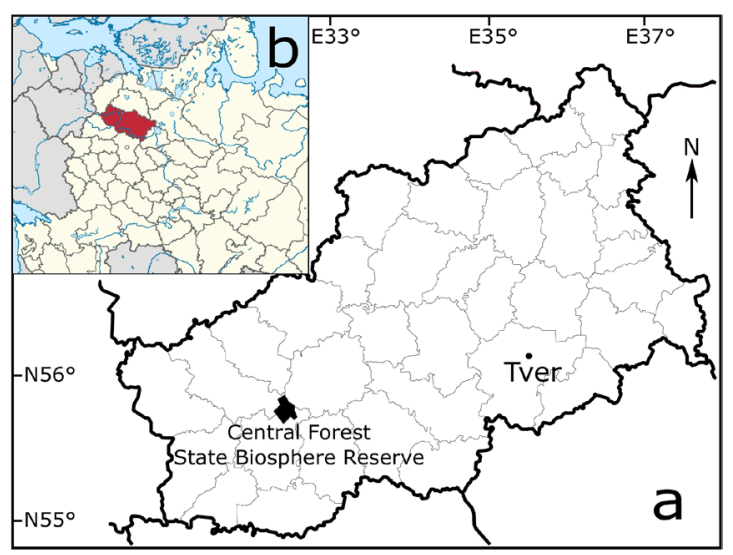

Fig. 1. Geographic location of the collecting area: a - the Central Forest State Biosphere Reserve in Tver region, b-Tver region in European Russia.

(approximately 1 ha each), so that each site was visited three or four times in different months. The study area is comprised of three windfalls of different ages (in 1960 (1), 1987 (2) and 1996 (3)) surrounded by old-growth spruce (Picea $a b$ ies (L.) H. Karst.) forests and four forest areas of different successional stages (two old-growth forests: green moss spruce-with aspen (Populus tremula L.) forest (4), herb spruce forest (5) and two young forest types: herb spruce, alder (Alnus incana (L.) Moench), elm (Ulmus laevis Pall.), lime (Tilia cordata Mill.) with birch (Betula pendula Roth) forest (6), herb birch forest (7) (Fig. 2). All sites harbour large amounts of dead wood except the younger forests $(6,7)$. Surveyed logs were chosen randomly according to their size (min. $5 \mathrm{~cm}$ in diameter). Fragments of each individual fruit body were collected for microscopic identification using 5\% $\mathrm{KOH}$, Melzer's reagent (IKI) and Cotton Blue (CB). In this paper we included findings of polypores and corticioid species, and our concept generally follows that of Bernicchia and Gorjón (2010). The list consists of Latin species name followed by substrate type (in some cases specified only up to angiosperm wood) and the number of collecting location. Numbered specimens deposited in personal fungarium can be requested from the Zoological Club of the Zoological Museum of Moscow University (KUN ZMMU). The specimens without number are deposited in personal fungarium of I.V. and H.K. The nomenclature follows mostly Kotiranta et al. (2009), Bernicchia \& Gorjón

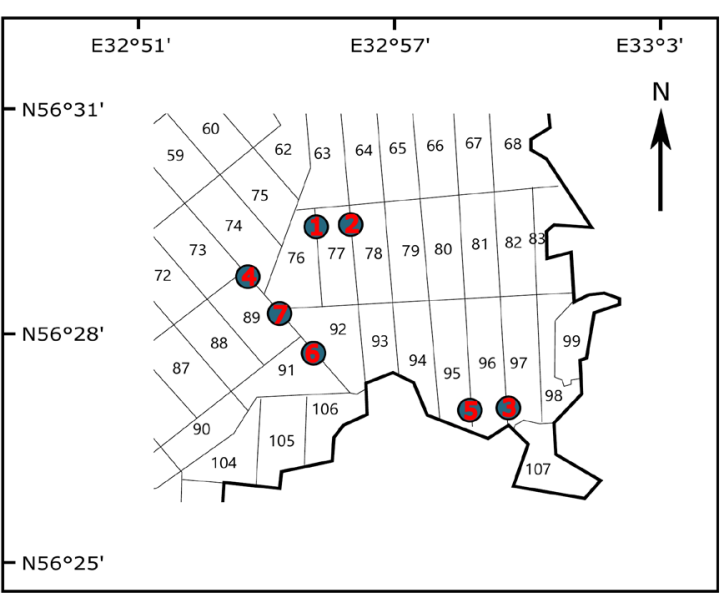

Fig. 2. The scheme of the south-eastern part of the Central Forest State Biosphere Reserve with collecting locations (in circles) and compartment numbers.

(2010) and Ryvarden \& Melo (2014). MycoBank was also consulted when species were missing from the listed sources. Infraspecific taxa were not specified.

\section{RESULTS AND DISCUSSION}

In total, 228 species of polyporoid and corticioid basidiomycetes were collected, of them 139 grew on $P$. abies and 128 on angiosperm logs. Most of the species were observed exclusively on gymnosperm or angiosperm wood. In particular, 99 species grew exclusively on $P$. abies and 89 on angiosperm wood, while only 40 were collected both from gymnosperms and angiosperms. The large spruces are known to inhabit more species than small ones (e.g. Renvall, 1995) and the sites (1-5) are thus favourable for many wood-inhabiting fungi (Berglund et al., 2011). At the same time, young forest sites $(6,7)$ with the highest abundance of angiosperm dead wood harbour no large logs at all.

We found 37 species which are new to the Central Forest Reserve (Kotkova, 2014b) and also registered five species included in the Red List of the Tver Region: Antrodiella foliaceodentata (Nikol.) Gilb. \& Ryvarden, Ganoderma lucidum (Curtis : Fr.) P. Karst., Hericium coralloides (Scop. : Fr.) Pers., Polyporus badius (Pers.) Schwein., Pycnoporellus fulgens (Fr.) Donk (Sorokin, 2002) of which H. coralloides is also 
included in the Red List of Russia (Trutnev, 2008). H. coralloides, P. badius and P. fulgens were frequently observed in our study sites, indicating that these are rather common in the reserve. This partly disagrees with their assignment to threat category 3 in the Red Book of the Tver region, because this category suggests that the species has a sporadic distribution (Sorokin, 2002; Trutnev, 2008). However, our study had a narrow geographic focus on one of the best preserved forests in the region. In addition, the threat category 1 applied to G. lucidum suggests that species faces a high risk of extinction (Sorokin, 2002) which we question since we found G. lucidum in both old-growth forest $(3,4)$ and young forest sites (6). One Phlebiella species is probably new to science indicating that studying fungal biodiversity has received only limited emphasis even in easily accessible areas of European Russia.

\section{Annotated list of species}

The species list is in alphabetical order. Asterisks $(*)$ indicate new records for the Central Forest Reserve. The entries consist of a scientific name followed by substrate species (when available), formatted personal fungarium specimen numbers (KUN ZMMU 1234) and number of the collecting location (Fig. 2). Short notes are provided for some records. As these records originate within a strictly protected area, we do not provide precise coordinates for the locations.

Alutaceodontia alutacea (Fr. : Fr.) Hjortstam \& Ryvarden - angiosperm wood, Picea abies (KUN ZMMU 528, 661, 943, 986, 1748); 1, $2,3,4,5$.

Amphinema byssoides (Pers. : Fr.) J. Erikss. angiosperm wood, Picea abies, Populus tremula, mosses (KUN ZMMU 1595, 1638, 1692, 1758, 1766, 1767, 1803, 1806, 1855); $1,2,3,6$.

*Amylocorticium cebennense (Bourdot) Pouzar Picea abies (KUN ZMMU 670); 1, 2.

Amylocorticium subincarnatum (Peck) Pouzar - Picea abies (KUN ZMMU 558, 642, 647, 1551, 1930); 1, 2, 3, 5.

Amylocorticium subsulPhureum (P. Karst.) Pouzar - Picea abies (KUN ZMMU 673); 3.

Amylocystis LaPPONICA (Romell) Singer - Picea abies (KUN ZMMU 43); 1, 2, 3, 4, 5, 6.

*Amylostereum chailletil (Pers. : Fr.) Boidin Picea abies (KUN ZMMU 665); 1, 3.
*Antrodia heteromorpha (Fr. : Fr.) Donk - Picea abies (KUN ZMMU 1928); 1.

ANTRODIA MACRA (Sommerf.) Niemelä - Populus tremula (KUN ZMMU 2079); 2.

AnTROdia PICEATA K. Runnel, V. Spirin \& J. Vlasák - Picea abies (KUN ZMMU 571, 583, 914, 920); 1, 2, 3, 4.

Notes - Earlier records of this (Kotkova, 2014b) were treated as Antrodia sitchensis (D. V. Baxter) Gilb. \& Ryvarden.

AnTROdia SERIALIS (Fr.) Donk - Picea abies (KUN ZMMU 55, 248, 251, 266, 286, 568); 1, 2, $3,4,5,6$.

Antrodia sinuosa (Fr.) P. Karst. - Picea abies (KUN ZMMU 52, 86, 249, 680, 689, 902, 964, 972); 1, 2, 3, 4, 5, 6.

Antrodia Xantha (Fr. : Fr.) Ryvarden - Picea abies (KUN ZMMU 273, 1932); 1, 2, 3.

ANTRODIELLA CITRINELla Niemelä \& Ryvarden - Picea abies (KUN ZMMU 2062); 2, 3.

ANTRODIELLA FAGINEA Vampola \& Pouzar - angiosperm wood, Populus tremula (KUN ZMMU 904, 1872); 1, 2, 4.

Antrodiella foliaceodentata (Nikol.) Gilb. \& Ryvarden - Populus tremula (KUN ZMMU 581); 6.

The find was made by J. Kinnunen in August 2014; this is the second report of this rare species in the reserve after Bondartseva (1986).

Antrodiella Pallescens (Pilát) Niemelä \& Miettinen - angiosperm wood (KUN ZMMU 907); $5,6,7$.

ANTRODiella ROMELli (Donk) Niemelä - angiosperm wood (KUN ZMMU 1825); 3.

ApHanobasidium PSEUdotsugae (BuRT) BoId. \& Gilles - Picea abies (KUN ZMMU 688, 1749); 3.

Asterodon fERruginosus Pat. - Picea abies (KUN ZMMU 1682); 2, 4, 5.

Athelia DECiPiens (Höhn. \& Litsch.) J. Erikss. angiosperm wood, Betula pendula, Picea $a b-$ ies, Populus tremula (KUN ZMMU 662, 949, 974, 977, 1523, 1539, 1550, 1575, 1582, 1643, 1678, 1704, 1711); 1, 2, 3, 4, 5, 6, 7.

Athelia EPIPHYLla Pers. : Fr. coll. - angiosperm wood, Betula pendula (KUN ZMMU 1621, 1622, 1667, 1870, 1871); 5, 7.

Notes - The concept of this species follows Eriksson and Ryvarden (1973).

Athelopsis GLAUCina (Bourdot \& Galzin) Parmasto - Picea abies (KUN ZMMU 521); 4. 
Athelopsis subinconspicuA (Litsch.) Jülich - Picea abies (KUN ZMMU 992, 1931); 5.

BASIDIODENDRON CINEREUM (Bres.) Luck-Allen Picea abies (KUN ZMMU 547); 5.

*BAsidiodendRon EYREI (Wakef.) Luck-Allen - angiosperm wood (KUN ZMMU 1654); 5.

*BASIDIODENDRON RADIANS (Rick) P. Roberts - angiosperm wood (KUN ZMMU 1664); 5.

Notes - We failed to find earlier reports of this species from Russia, thus it might represent a new species for the country. Finding this species was expected, however, since it has been collected in Estonia (Sell \& Kotiranta 2011) and Finland (Kotiranta \& Saarenoksa 2005).

Basidioradulum Radula (Fr.) Nobles - Populus tremula (KUN ZMMU 1510); 4.

BJERKANDERA ADUSTA (Willd : Fr.) P. Karst. - angiosperm wood, Populus tremula (KUN ZMMU 1616, 1629, 1850); 1, 2, 3, 4, 5, 6, 7.

*BJerkandera fumosa (Pers. : Fr.) P. Karst. angiosperm wood, Populus tremula (KUN ZMMU 2226); 1, 2, 3, 4, 6, 7.

BoIdinia FURFURACEA (Bres.) Stalpers \& Hjortstam - Populus tremula (KUN ZMMU 1826); $1,2,3$.

Botryobasidium aureum Parmasto - Picea $a b$ ies; 2.

BOTRYOBASIDIUM BOTRYOSUM (Bres.) J. Erikss. - angiosperm wood, Picea abies, Populus tremula (KUN ZMMU 545, 549, 551, 643, 657, 678, 947, 955, 961, 962, 963, 968, 998, 1512, $1548,1565,1659,1663,1690,1696,1697$, $1699,1754,1757,1813,1820,1827,1841$ ); $1,2,3,4,5$.

Botryobasidium CANDICANS J. Erikss. - angiosperm wood, Picea abies (KUN ZMMU 992, 1824); 1, 2, 3, 5.

*Botryobasidium conspersum J. Erikss. - angiosperm wood, Picea abies, Populus tremula (KUN ZMMU 674, 681, 690, 1814, 1864); $1,3$.

BOTRYOBASIDIUM INTERTEXTUM (Schwein.) Jülich \& Stalpers - angiosperm wood (KUN ZMMU 1709, 1763); 3.

Botryobasidium ISABELlinum (Fr.) D.P. Rogers angiosperm wood, Picea abies (KUN ZMMU 538, 539, 556, 557, 649, 655, 672, 985, $1660,1707,1713,1753,1762,1763,1764$, 1792, 1793, 1830, 1832, 1848); 1, 2, 3, 4, 5.

Botryobasidium MEdium J. Erikss. - Picea abies (KUN ZMMU 534, 685, 959, 996); 5.
Botryobasidium obtusisporum J. Erikss. - Picea abies (KUN ZMMU 652, 976, 1545, 1556, 1689, 1732); 1, 2, 3, 4, 5.

BOTRYOBASIDIUM SUBCORONATUM (Höhn. \& Litsch.) Donk - angiosperm wood, Betula pendula, Picea abies (KUN ZMMU 526, 531, 535, 537, 544, 550, 553, 554, 644, 666, 671, 676, 939, $940,950,952,956,958,967,1557,1569$, $1578,1579,1585,1587,1589,1596,1597$, $1599,1604,1630,1631,1634,1635,1687$, $1706,1714,1721,1727,1729,1750,1800$, 1801, 1823, 1831); 1, 2, 3, 4, 5, 6, 7.

${ }^{*}$ CERACEOMYCES MICROSPORUS K.H. Larss. - angiosperm wood (KUN ZMMU 1593); 5.

Ceraceomyces tessulatus (W.B. Cooke) Jülich angiosperm wood (KUN ZMMU 1657); 3, 5.

CERACEOMYCES VIOLASCENS (Fr. : Fr.) Jülich - Picea abies; 3.

CERIPORIA EXCELSA (S. Lundell) Parmasto - angiosperm wood (KUN ZMMU 903); 5, 6.

CERIPORIOPSIS ANEIRINA (Sommerf. : Fr.) Domański - Populus tremula; 6.

CERIPORIOPSIS MUCida (Pers. : Fr.) Gilb. \& Ryvarden - angiosperm wood (KUN ZMMU 979); 5.

CERIPORIOPSIS SUBVERMISPORA (Pilat) Gilb. \& Ryvarden - Betula pendula, Picea abies (KUN ZMMU 594, 1798); 3.

CERRENA UniColor (Bull. : Fr.) Murrill - angiosperm wood (KUN ZMMU 1668); 1, 2, 3, 4, $5,6,7$.

Chondrostereum purpureum (Pers. : Fr.) Pouzar - angiosperm wood; 6, 7.

Cinereomyces lindBladi (Berk.) Jülich - angiosperm wood, Picea abies (KUN ZMMU 51, 53, 54, 100, 108, 246, 250, 269, 271, 287, 1628); 3, 4, 6.

Climacocystis borealis (Fr.) Kotl. \& Pouzar - Picea abies (KUN ZMMU 90); 1, 2, 3.

Conferticium ochraceum (Fr. : Fr.) Hallenb. Picea abies; 3.

Coniophora ARIDA (Fr.) P. Karst. - Picea abies (KUN ZMMU 1603, 1807); 1, 2, 3, 4, 5, 6.

Coniophora olivacea (Pers. : Fr.) P. Karst. - Picea abies (KUN ZMMU 105); 1, 2, 3.

Coniophora PUTEANA (Schumach. : Fr.) P. Karst. Picea abies (KUN ZMMU 664, 955); 1, 2, 5.

${ }^{*}$ CRistinia helvetica (Pers.) Parmasto - angiosperm wood (KUN ZMMU 1795); 3.

Crustoderma dryinum (Berk. \& M.A. Curtis) Parmasto - Picea abies (KUN ZMMU 552, 942, 960); 1, 2, 5 .

Cytidia salicina (Fr.) Burt - Salix caprea (KUN ZMMU 1676); 7. 
Dacryobolus sudans (Fr.) Fr. - Picea abies; 2.

DAEDALEOPSIS CONFRAGOSA (Bolton : Fr.) J. Schröt. - angiosperm wood; 1, 2, 3, 4, 5, 6, 7 .

DAEDALEOPSIS SEPTENTRIONALIS (P. Karst.) Niemelä - Betula pendula (KUN ZMMU 1624); 1, 2, $3,4,5,6,7$.

DAEDAleopsis TRICOLOR (Bull.) Bondartsev \& Singer - angiosperm wood (KUN ZMMU 1627, 1671); 1, 2, 3, 4, 6.

Datronia MOLLIS (Sommerf. : Fr.) Donk - Populus tremula (KUN ZMMU 1849); 1, 2, 3, 4, 6, 7.

DentiPELLIS FRaGilis (Pers.: Fr.) Donk - angiosperm wood (KUN ZMMU 593, 1789); 4, 5.

Dichostereum boreale Pouzar - Picea abies; 1 , $2,3$.

*Exidia Pithya (Alb. \& Schwein. : Fr.) Fr. - Picea abies; 1, 2, 3, 4, 5, 6.

EXIDIOPSIS UMBRINA (D.P. Rogers) Wojewoda - Picea abies; 1.

*FibRicium RUdE (P. Karst.) Jülich - angiosperm wood (KUN ZMMU 909); 4.

FOMES FOMENTARIUS (L. : Fr.) J. Kickx - Betula pendula (KUN ZMMU 1684); 1, 2, 3, 4, 5, 6, 7.

Fomitopsis Pinicola (Sw. : Fr.) P. Karst. - angiosperm wood, Picea abies (KUN ZMMU 103, 901); 1, 2, 3, 4, 5, 6, 7 .

Fomitopsis Rosea (Alb. \& Schwein. : Fr.) P. Karst Picea abies (KUN ZMMU 48); 1, 2, 3, 4, 5, 6.

Ganoderma APPlanatum (Pers.) Pat. - angiosperm wood, Tilia cordata (KUN ZMMU 42); 1, 2, $3,4,5,6,7$.

Ganoderma Lucidum (Curtis : Fr.) P. Karst. - angiosperm wood; $3,4,6$.

GLOEOCYSTIDIELLUM POROSUM (Berk. \& M.A. Curtis) Donk - angiosperm wood, Picea abies (KUN ZMMU 675, 965); 7.

Gloeopeniophorella convolvens (P. Karst.) Boidin, Lanq. \& Gilles - angiosperm wood, Picea abies, Populus tremula (KUN ZMMU 555, 648, 989, 1511, 1512, 1514, 1517, 1533, 1572, 1612, 1673, 1841, 1857); 1, 2, $3,4,5,6,7$.

Gloeophyllum abietinum (Bull. : Fr.) P. Karst.Picea abies; 2.

GLOEOPHYLLUM OdORATUM (Wulfen : Fr.) Imazeki Picea abies; 1, 2, 3, 4, 5, 6.

Gloeophyllum SEPIARIum (Wulfen : Fr.) P. Karst. - Picea abies (KUN ZMMU 247, 272, 953, 970); 1, 2, 3, 4, 5, 6.

Gloeoporus Dichrous (Fr. : Fr.) Bres. - Betula pendula (KUN ZMMU 46); 1, 2, 3, 4, 5, 6.

Gloeoporus PANNOCINCTUS (Romell) J. Erikss. angiosperm wood (KUN ZMMU 590); 4, 5.
Hapalopilus Rutilans (Pers.) Murrill - Populus tremula (KUN ZMMU 1544); 1, 2, 3, 4, 6, 7. Hericium CORAlloides (Scop. : Fr.) Pers. - angiosperm wood (KUN ZMMU 118 ); 2, 3.

HETEROBASIDION PARVIPORUM Niemelä \& Korhonen - Picea abies (KUN ZMMU 245); 1, 2, 3, 4, 5.

Hymenochaete tabacina (Sowerby) Lév - angiosperm wood (KUN ZMMU 530, 1836); 1, 2, $3,4,5,6,7$.

Hyphoderma ARgillaceum (Bres.) Donk - angiosperm wood, Picea abies (KUN ZMMU 975, 1637, 1646, 1702, 1753); 4, 6.

Hyphoderma CREMEOAlBUm (Höhn. \& Litsch.) Jülich - Picea abies (KUN ZMMU 1861); 3.

HyPHODERMA LITSCHAUERI (Burt) J. Erikss. \& Å. Strid- angiosperm wood, Betula pendula, Picea abies, Populus tremula (KUN ZMMU 543, 1516, 1584, 1594, 1710, 1856); 4, 5, 7.

Hyphoderma SETIGERUM (Fr.) Donk - angiosperm wood, Betula pendula, Populus tremula (KUN ZMMU 1541, 1581, 1586, 1590, 1619, 1708); 1, 2, 3, 4, 7 .

HypHOdontia AluTARIA (Burt) J. Erikss. - angiosperm wood, Picea abies, Populus tremula (KUN ZMMU 529, 994, 1518, 1542, 1543, 1560, 1645, 1838); 1, 2, 3, 4, 5, 6.

HyPHODONTIA PALLIDUla (Bres.) J. Erikss. - angiosperm wood, Picea abies, Populus tremula (KUN ZMMU 1577, 1610, 1686, 1693, 1719, 1723, 1725, 1787, 1804, 1815); 1, 2, 3, 4, 6.

*Hypochnicium CREMicolor (Bres.) H. Nilsson \& Hallenb. - Populus tremula (KUN ZMMU 645, 1833); 3.

HYPOCHNICIUM SUBRIGESCENS Boidin - angiosperm wood (KUN ZMMU 1652); 4, 5.

*Hypochnicium vellereum (Ellis \& Cragin) Parmasto - Populus tremula (KUN ZMMU 1672); 5.

HyPOCHNICIUM WAKEFIELDIAE (Bres.) J. Erikss. - angiosperm wood, Picea abies, Populus tremula (KUN ZMMU 646, 668, 1527, 1658, 1688, $1695,1759,1760,1816,1819,1837,1844)$; $1,2,3,4,5$.

InoNotus obliQuUs (Pers. : Fr.) Pilát - Alnus incana, Betula pendula; 1, 2, 3, 4, 5, 6 .

Notes - Only sterile conks were observed.

InONOTUS RADIATUS (Sowerby : Fr.) P. Karst. - angiosperm wood; 1, 2, 3, 4, 5, 6, 7 .

Inonotus RheAdes (Pers.) P. Karst. - Populus tremula (KUN ZMMU 2130); 4.

IschNOdERMA BENZOINUM (Wahlenb. : Fr.) P. Karst. - Picea abies; 2, 3. 
Junghuhnia collabens (Fr.) Ryvarden - Picea $a b$ ies (KUN ZMMU 87, 275, 281, 911, 1934); $1,2,3,4,5$.

JunghuHNia LACERA (P. Karst.) Niemelä \& Kinunnen - Populus tremula (KUN ZMMU 592); 2,4 .

Junghuhnia LuteoAlba (P. Karst.) Ryvarden $-P i-$ cea abies (KUN ZMMU 560, 1810); 1, 2, 3.

JunghuHNiA NITIDA (Pers . : Fr.) Ryvarden - Alnus incana, Populus tremula (KUN ZMMU 36, 1854); 6.

Kneiffiella BARBA-Jovis (Bull. : Fr.) P. Karst. Populus tremula (KUN ZMMU 1519, 1520, 1522); 4.

KNEIFFIELLA CINERACEA (Bourdot \& Galzin) Jülich \& Stalpers - Picea abies (KUN ZMMU 667); 3.

KNeIFfiella subalutacea (P. Karst.) Jülich \& Stalpers - angiosperm wood, Populus tremula (KUN ZMMU 1535, 1639, 1666, 1712, 1769, 1821); 1, 2, 3, 4, 5, 6.

LAETIPORUS SUlPhUREUS (Bull. : Fr.) Murrill coll. - Picea abies; 6.

Notes - Unfortunately, the specimen was poorly dried and unsuitable for microscopic identification. There was only one collection of Laetiporus from spruce in the reserve and this might represent $L$. montanus Černý ex Tomšovský \& Jankovský which is restricted to coniferous wood. It has been reported from central European mountain forests (Tomsovský \& Jankovský, 2008) and at least from the Republic of Tuva in southern Siberia (Kotiranta et al., 2016) plus Jewish Autonomous Region in Russian Far East (Bukharova \& Zmitrovich, 2014).

LaXiteXTUm BICOLOR (Pers. : Fr.) Lentz - angiosperm wood (KUN ZMMU 1867); 3.

LEPTOPORUS MOLLIS (Pers. : Fr.) Quél. - Picea abies (KUN ZMMU 41, 180, 1731); 1, 2, 3.

LEPTOSPOROMYCES GALZINII (Bourdot) Jülich - Picea abies; 1.

Leucogyrophana mollusca (Fr.) Pouzar - Picea abies, Populus tremula (KUN ZMMU 684, 951, 963, 971, 1525, 1555); 1, 2, 3, 4, 5.

Leucogyrophana Romelli (Fr.) Ginns - Populus tremula (KUN ZMMU 1521); 4.

Metulodontia nivea (P. Karst.) Parmasto - Picea abies; 1.

Mucronella calva (Alb. \& Schwein.) Fr. - Picea abies (KUN ZMMU 640); 1, 2, 3, 4, 5, 6.

Oligoporus ALNi (Niemelä \& Vampola) Piiitek angiosperm wood; $1,2,3,4,5,6,7$.
Oligoporus caesius (Schrad.: Fr.) Gilb. \& Ryvarden - Picea abies (KUN ZMMU 102, 1567, 1601, 1845); 1, 2, 3, 4, 5, 6.

Oligoporus FRAGILIS (Fr.) Gilb. \& Ryvarden - Picea abies (KUN ZMMU 106, 113, 561, 566, 1568, 1808); 1, 2, 3, 4, 5 .

Oligoporus GUTTULATUS (Peck) Gilb. \& Ryvarden - Picea abies (KUN ZMMU 99); 2, 3.

Oligoporus leucomallellus (Murrill) Gilb. \& Ryvarden - Picea abies; 2.

Oligoporus Placentus (Fr.) Gilb. \& Ryvarden - Picea abies (KUN ZMMU 61, 475, 584, 637); 1.

Oligoporus PTYCHOGASTER (F. Ludw.) Falck \& O. Falck - Picea abies; 2.

*Oligoporus Rennyi (Berk. \& Broome) Donk - Picea abies (KUN ZMMU 1937); 2.

*Oligoporus Romellit (M. Pieri \& B. Rivoire) Niemelä - Picea abies; 1, 2.

OLIGOPORUS SERICEOMOLLIS (Romell) Bondartseva - Picea abies; 1, 2, 3.

Oligoporus tephroleucus (Fr.) Gilb. \& Ryvarden - angiosperm wood, Picea abies (KUN ZMMU 97, 98, 101, 1592, 1834); 1, 2, 3, 4, 5, 6, 7.

Oligoporus undosus (Peck) Gilb. \& Ryvarden angiosperm wood, Picea abies (KUN ZMMU 95, 532, 1786); 1, 2, 3, 4, 5, 6, 7.

OliveONIA FIBRILLOSA (Burt) Donk - Picea abies; 1.

OXYPORUS CORTICOLA (Fr.) Ryvarden - angiosperm wood, Picea abies, Populus tremula (KUN ZMMU 35, 89, 900, 1605, 1656, 1665, 1859); 2.

OXYPORUS POPULINUS (Schumach. : Fr.) Donk angiosperm wood, Populus tremula (KUN ZMMU 981, 1829); 1, 2, 3, 4, 5, 6.

*Peniophorella Pallida (Bres.) K.H. Larss. - angiosperm wood, Picea abies (KUN ZMMU 524, 546, 941, 951, 980, 1679); 1, 2, 3, 4, $5,6$.

Peniophorella praetermissa (P. Karst.) K.H. Larss. - Picea abies, Populus tremula (KUN ZMMU 533, 536, 679, 957, 1538, 1562, 1613); 1, 2, 3, 4, 5 .

PENIOPHORELla PUBERA (Fr.) P. Karst. - angiosperm wood, Betula pendula, Picea abies, Populus tremula (KUN ZMMU 978, 984, 1515, 1524, $1588,1620,1632,1684,1809,1843) ; 1,2$, $3,4,5,6,7$.

*Perenniporia medulla-Panis (Jacq. : Fr.) Donk Tilia cordata (KUN ZMMU 302); 2.

PERENNIPORIA SUbACIDA (Peck) Donk - Picea abies (KUN ZMMU 38, 109, 112, 268, 304, 305, 1822); 1, 2, 3, 4, 5 . 
Phanerochaete laevis (Pers. : Fr.) J. Erikss. \& Ryvarden - Picea abies; 1.

*Phanerochaete sordida (P. Karst.) J. Erikss. \& Ryvarden - angiosperm wood, Picea abies (KUN ZMMU 1633, 1858); 6.

Phellinus ABIETIS (P. Karst.) Jahn - Picea abies (KUN ZMMU 96); 1, 2.

Notes - Earlier collections of this were probably treated as Phellinus chrysoloma (Fr.) Donk sensu lato (Kotkova, 2014b).

Phellinus ferrugineofuscus (P. Karst.) Bourdot - Picea abies; 1, 2, 3, 4, 5.

Phellinus laevigatus (Fr.) Bourdot \& Galzin Betula pendula; 3.

Phellinus nigrolimitatus (Romell) Bourdot \& Galzin - Picea abies (KUN ZMMU 56, 92, 107, 114, 274, 496, 954, 995); 1, 2, 3, 4, 5.

Phellinus PUNCTATUS (P. Karst.) Pilát - angiosperm wood (KUN ZMMU 982); 1, 2, 3, 4, 5, 6, 7.

Phellinus viticola (Schwein. in Fr.) Donk - Picea abies (KUN ZMMU 49, 119, 1700, 1747); 1, 2, 3, 4, 5 .

Phlebia Centrifuga P. Karst. - Picea abies (KUN ZMMU 1929); 1, 2, 3, 4, 5.

*Phlebia firma J. Erikss. \& Hjortstam - Picea abies (KUN ZMMU 659); 2.

Phlebia gigantea (Fr. : Fr.) Donk - Picea abies (KUN ZMMU 520, 966, 997); 1, 2, 3, 4, 5, 7.

Phlebia Lilascens (Bourdot) J. Erikss. \& Hjortstam - Picea abies; 1, 2.

Phlebia livida (Pers. : Fr.) Bres. - Picea abies (KUN ZMMU 1547); 1, 5.

Phlebia Radiata Fr. - angiosperm wood, Populus tremula (KUN ZMMU 983, 1614, 1677, 1852); 4, 5, 6.

Phlebia RUfa (Pers. : Fr.) M.P. Christ. - angiosperm wood (KUN ZMMU 1669); 5.

Phlebia subulata J. Erikss. \& Hjortstam - Picea abies (KUN ZMMU 1936); 2.

Phlebia TRemellosa (Schrad. : Fr.) Nakasone Populus tremula; 3, 6, 7.

*Phlebiella borealis K.H. Larss. \& Hjortstam Picea abies (KUN ZMMU 1752); 3.

*Phlebiella fibrillosa (Hallenb.) K.H. Larss. \& Hjortstam - angiosperm wood (KUN ZMMU 1817); 3.

Notes - This is the second find of this rare species in Russia according to the available literature; previously it was found in the Jewish Autonomous Region in the Russian Far East (Bukharova \& Zmitrovich, 2014).
Phlebiella sp. - Picea abies (KUN ZMMU 1851); 3.

Notes - Basidiocarp thin, pruinose, resupinate. Hymenophore smooth and grey. Hyphal system monomitic; all hyphae clamped, thin-walled 2-2.5 $\mu \mathrm{m}$ wide, sinuous. Cystidia absent. Basidia pleural, cylindrical, with four sterigmata, 8-13 × 4-6 $\mu \mathrm{m}$, containing few or no oily droplets. Basidiospores subglobose to globose, warted (2.5-)3.3-3.4 $\times(2.9-) 3.1-3.2 \mu \mathrm{m}$, negative in IKI and CB (Fig. 3).

This species was found only once on a spruce log and its identification is problematic. It is probably an undescribed species within Phlebiella sensu stricto because it clearly resembles the core species of this genus. Our specimen has clearly smaller spores than Phlebiella ardosiaca (Bourdot \& Galzin) K.H. Larss. \& Hjortstam (3.3-3.4 $\times 3.1-3.2$ vs $5-6 \mu \mathrm{m}$ ) which also has globose basidiospores. Other European species of Phlebiella are clearly separated by their spore shape. Further studies are needed to confirm the identity and status of this taxon.

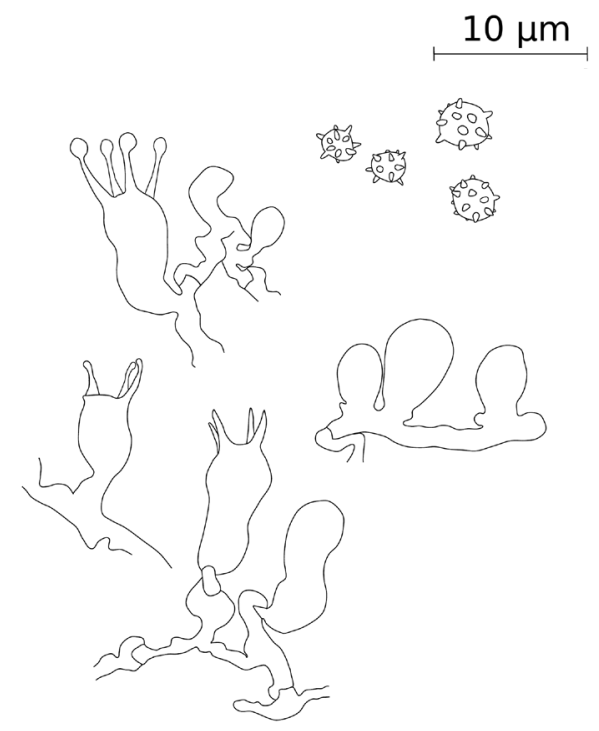

Fig. 3. Hyphae, basidia and spores of Phlebiella sp. (KUN ZMMU 1851).

Phlebiella sulphurea (Pers. : Fr.) Ginns \& M.N.L. Lefebvre - angiosperm wood, Picea abies, Populus tremula (KUN ZMMU 669, 687, 1532, 1609, 1611, 1661, 1662, 1705); 1, $2,3,4,5$. 
Physisporinus sanguinolentus (Alb. \& Schwein. : Fr.) Pilát - angiosperm wood (KUN ZMMU 2060); 3, 6.

Piloderma Byssinum (P. Karst.) Jülich - angiosperm wood (KUN ZMMU 1715); 1, 2.

Piloderma fallax (Liberta) Stalpers - Populus tremula (KUN ZMMU 1513, 1543, 1608); 4.

Plicatura Nivea (Sommerf. : Fr.) P. Karst. - Betula pendula, Populus tremula (KUN ZMMU 1580, 1839); 7.

POLYPORUS BADIUS (Pers.) Schwein. - angiosperm wood (KUN ZMMU 1853); 3, 5.

POLYPORUS VARIUS Pers. : Fr. - Populus tremula (KUN ZMMU 91); 2, 3.

Pseudohydnum gelatinosum (Scop. : Fr.) P. Karst. - Picea abies; 2, 3.

Pseudomerulius Aureus (Fr.) Jülich - Picea abies (KUN ZMMU 1805); 3.

Punctularia STRIgosozonata (Schwein.) Talbot - Populus tremula (KUN ZMMU 748); 5, 6.

Pycnoporellus Alboluteus (Ellis \& Everh.) Kotl. \& Pouzar - Picea abies (KUN ZMMU 37, 179, 498); 2, 3.

Pycnoporellus fulgens (Fr.) Donk - angiosperm wood, Picea abies (KUN ZMMU 40, 50, 582, 1626); 1, 2, 3, 4, 5 .

RePETOBASIDIUM MIRIFICUM J. Erikss. - Picea $a b-$ ies; 2.

Resinicium BICOLOR (Alb. \& Schwein. : Fr.) Parmasto - angiosperm wood, Picea abies, Populus tremula (KUN ZMMU 527, 944, $1509,1576,1600,1607,1641,1691)$; 1 , $2,3,4,5,6$.

Resinicium furfuraceum (Bres.) Parmasto - Picea abies; $1,3$.

Rigidoporus CROcatus (Pat.) Ryvarden - Picea abies; 6.

*SARCoporia Polyspora P. Karst. - Picea abies (KUN ZMMU 73, 122, 178, 910); 1, 2, 3, 4, 5.

*Schizopora flavipora (BERK. \& M.A. Curtis) RYvARDEN - Betula pendula (KUN ZMMU 588); $1,2,3$.

*SCOPUlOIDES RIMOSA (W.B. Cooke) Jülich - angiosperm wood (KUN ZMMU 988); 6.

Scytinostroma Galactinum (Fr.) Donk - angiosperm wood, Picea abies (KUN ZMMU 990, 991); 5.

Serpula himantioides (Fr. : Fr.) P. Karst. - Picea abies (KUN ZMMU 651); 1, 2.

SistoTREMA BRINKMANNII (Bres.) J. Erikss. - angiosperm wood, Picea abies, Populus tremula (KUN ZMMU 1680, 1683, 1716, 1812, 1828, 1863, 1865); 5.
Sistotrema Raduloides (P. Karst.) Donk - Populus tremula (KUN ZMMU 912); 5.

SistoTREMA RESINICYSTIDIUM Hallenb. - Picea abies (KUN ZMMU 650); 1.

SistoTREMASTRUM SUECICUM Litsch. ex J. Erikss. Picea abies (KUN ZMMU 683, 1799, 1868); 2, 3.

Skeletocutis Amorpha (Fr.) Kotl. \& Pouzar - Picea abies (KUN ZMMU 104, 110, 111, 1728); $1,2,3$.

Skeletocutis BREVISPORA Niemelä - Picea abies (KUN ZMMU 908); 5.

Skeletocutis Kuehneri A. David - Picea abies (KUN ZMMU 499, 1736); 1, 3.

Skeletocutis nivea (Jungh.) Jean Keller - angiosperm wood, Corylus avellana (KUN ZMMU 121, 589); 1, 2, 3, 4, 5, 6, 7 .

Skeletocutis OdORA (Sacc.) Ginns - Picea abies, Populus tremula (KUN ZMMU 282, 283, 284, 285, 567, 591, 899, 919, 973, 999); $1,2,3,4,5$.

Skeletocutis Papyracea A. David - Picea abies (KUN ZMMU 559, 906, 913); 4, 5.

StecCherinum FImBRiatum (Pers. : Fr.) J. Erikss. angiosperm wood, Betula pendula, Populus tremula (KUN ZMMU 1591, 1623, 1715); 4, 7 .

*STECCHERINUm MURASHKInSKYI (Burt) Maas Geest. - angiosperm wood (KUN ZMMU 1574, 1647); 6.

*Stereum gausapatum (Fr.) Fr. - angiosperm wood; 6.

Stereum hiRsutum (Willd. : Fr.) Pers. - angiosperm wood, Populus tremula (KUN ZMMU 1617, 1625, 1642); 1, 2, 3, 4, 6.

SteREum RUGOSUm Pers. : Fr. - angiosperm wood; 6,7 .

Stereum sanguinolentum (Alb. \& Schwein. : Fr.) Fr. - Picea abies; 1, 2, 3.

Stereum subtomentosum Pouzar - angiosperm wood; 3, 6.

*Thanatephorus Fusisporus (J. Schröt.) P. Roberts \& Hauerslev - Picea abies; 1, 3.

ThelePhora PALMATA Scop. : Fr. - angiosperm wood (KUN ZMMU 585); 5.

Trametes Betulina (L. : Fr.) Pilát - Betula pendula (KUN ZMMU 44, 1670); 1, 2, 3, 5, 6, 7.

TRAMETES GiBBOSA (Pers.) Fr. - angiosperm wood, Populus tremula; 1, 2, 3, 4, 5, 6 .

TRAMETES hiRSUTA (Fr.) Pilát - angiosperm wood (KUN ZMMU 2058); 1, 2, 3, 4, 5, 6.

Trametes ochracea (Pers.) Gilb. \& Ryvarden angiosperm wood (KUN ZMMU 2061); 1, 2, $3,4,5,6,7$. 
Trametes pubescens (Schumach. : Fr.) Pilát angiosperm wood, Picea abies (KUN ZMMU 1933); 3.

Notes - The specimen KUN ZMMU 1933 grew on a spruce log which is an unusual substrate for T. pubescens (Ryvarden \& Melo, 2014).

TRAmetes TROGil Berk. in Trog. - angiosperm wood; 3, 6.

TRAmetes versicolor (L. : Fr.) Lloyd- Populus tremula (KUN ZMMU 1540); 1, 2, 3, 4.

TRECHISPORA COHAERENS (Schwein.) Jülich \& Stalpers - Betula pendula, Picea abies (KUN ZMMU 660, 677, 1561, 1583); 3, 5, 7.

TRECHISPORA FARINACEA (Pers. : Fr.) Liberta - angiosperm wood, Picea abies, Populus tremula (KUN ZMMU 522, 541, 1536, 1606, 1615, $1698,1724,1730,1755,1756,1802,1847)$; $1,2,3,4,5$.

*Trechispora hymenocystis (Berk. \& Broome) K.H. Larss. - Picea abies, Populus tremula (KUN ZMMU 523, 540, 969, 1531, 1537, 1554, 1846); 1, 2, 3, 4, 5.

*TRECHISPORA cf. MINIMA K.H. Larss. - Picea abies (KUN ZMMU 1703); 5.

Notes - Basidiocarp thin, resupinate. Hymenophore smooth, farinose, whitish to ochraceous. Hyphal system monomitic; all hyphae clamped without crystals. Subicular hyphae thin- to clearly thick-walled (2)3-4 $\mu \mathrm{m}$ wide often with ampullate septa, subhymenial hyphae broad, frequently shortcelled, branched, sometimes inflated (2)4$8(10) \mu \mathrm{m}$ in diam. Cystidia absent. Basidia terminal, cylindrical, with four sterigmata, $10-14 \times 4-5 \mu \mathrm{m}$, contents with few or no oily droplets. Basidiospores ellipsoid to subglobose, warted $+2.6-2.8(-3.5) \times 2-2.5(-3.4)) \mu \mathrm{m}$ including the aculei, hilar appendage only occasionally seen, positive in $\mathrm{CB}$, negative in IKI. Individual aculei rather long 0.3-0.6 $\mu \mathrm{m}$ (Fig 4.).

Our specimen seems to be close to Trechispora minima which, however has larger spores, viz. 3.5-4 × 3.2-3.7 $\mu \mathrm{m}$ and mostly a less prominent hilar appendage. Nevertheless, further studies are needed to confirm the status of this taxon.

TRECHISPORA SUBSPHAEROSPORA (Litsch.) Liberta Picea abies (KUN ZMMU 1726, 1768, 1851); 3.

TRICHAPTUM ABIETINUM (Dicks. : Fr.) Ryvarden Picea abies (KUN ZMMU 270); 1, 2, 3, 4, 5.
TRICHAPTUM BIFORME (Fr. in Klotzsch) Ryvarden Betula pendula; 1, 2, 3, 4, 5, 6 .

TRICHAPTUM FUSCOVIOLACEUM (Ehrenb. : Fr.) Ryvarden - Picea abies; 1, 2, 3.

*Tubulicrinis accedens (Bourdot \& Galzin) Donk - Picea abies (KUN ZMMU 987, 1602); 5, 6.

*Tubulicrinis Borealis J. Erikss. - Picea abies (KUN ZMMU 658); 2.

*Tubulicrinis Calothrix (Pat.) Donk - Picea abies (KUN ZMMU 1558); 5.

Tubulicrinis Glebulosus (Bres.) Donk - angiosperm wood (KUN ZMMU 1717, 1791); 1.

*TUBULICRINIS PROPINQUUS (Bourdot \& Galzin) Donk - Picea abies (KUN ZMMU 1598); 6.

TUBULICRINIS STRANGUlatus K.H. Larss. \& Hjortstam - Picea abies (KUN ZMMU 653, 945, 1563); $1,5$.

Tubulicrinis subulatus (Bourdot $\&$ Galzin) Donk - Picea abies; 2.

TUBULICRINOPSIS ELLIPSOSPORA Kotir., Hjortstam \& M. Kulju - Picea abies; 2.

*TULASNELLA EICHLERIANA Bres. - angiosperm wood (KUN ZMMU 1862); 3.

Notes - This species was expected since it seems to be widespread in Eurasia (Raitviir, 1967; Roberts, 1994; Roberts \& Hauerslev,

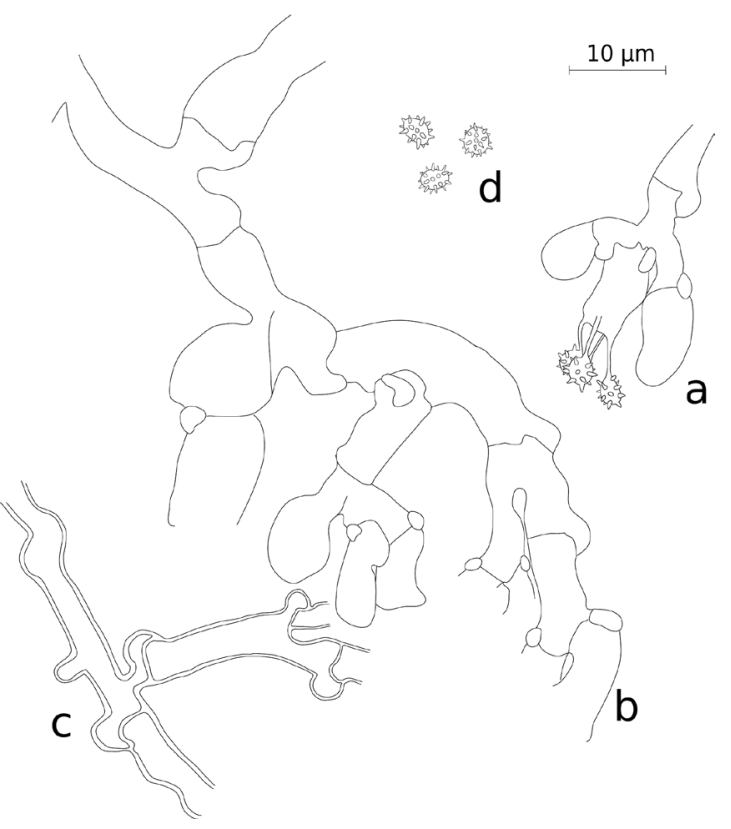

Fig. 4. Trechispora cf. minima (KUN ZMMU 1703): a) basidia b) subhymenial hyphae c) subicular hyphae d) spores. 
1997); in Russia it has been collected in the Sverdlovsk Region (Shiryaev et al., 2010) and Komi Republic (Viner, 2015).

TYLOSPORA ASTEROPHORA (Bonord.) Donk - Populus tremula (KUN ZMMU 1529); 4.

TYLOSPORA FIBRILLOSA (Burt) Donk - angiosperm wood, Picea abies (KUN ZMMU 1648, 1797); 5.

TYRomyces Fissilis (Berk. \& M.A. Curtis) Donk Populus tremula (KUN ZMMU 905); 6.

*VARARIa RACEMOSa (Burt) Rog. \& H.S. Jacks. Picea abies (KUN ZMMU 1935); 3.

*Vesiculomyces citrinus (Pers.) E. Hagstr. - angiosperm wood, Picea abies (KUN ZMMU 682, 686, 1549, 1552, 1553, 1564, 1674, 1675, 1811, 1860); 3, 5, 6.

Vuilleminia comedens (Nees. : Fr.) Maire - angiosperm wood (KUN ZMMU 1751); 6.

Xylodon Asperus (Fr.) Hjortstam \& Ryvarden - angiosperm wood, Picea abies, Populus tremula (KUN ZMMU 548, 747, 946, 948, $993,1526,1528,1530,1534,1546,1559$, $1566,1570,1571,1636,1640,1653,1655$, $1694,1722,1761,1790,1792,1818,1842)$; $1,2,3,4,5$.

XYLODON BREvisetus (P. Karst.) Hjortstam \& Ryvarden - angiosperm wood, Picea abies (KUN ZMMU 525, 956, 1644, 1649, 1650, $1685,1701,1714,1720,1869) ; 1,2,3,4$, $5,6$.

*Xylodon bugellensis (Ces.) Hjortstam \& Ryvarden sensu Bernicchia and Gorjón (2010) Populus tremula (KUN ZMMU 1840); 3.

Xylodon crustosus (Pers.: Fr.) Chevall - angiosperm wood, Picea abies (KUN ZMMU 1681, 1835); 6.

Xylodon spathulatus (Schrad. : Fr.) Kuntze Picea abies; 2.

\section{ACKNOWLEDGEMENTS}

We would like to thank Viacheslav A. Spirin, Tuomo Niemelä and Vera M. Kotkova for helpful discussions on the identification of some corticioid and polypore species, an anonymous reviewer for valuable comments on the manuscript, and Juha Kinnunen for providing additional specimens. We are grateful for the warm hospitality and support from the Central Forest State Biosphere Nature Reserve. I.V. was supported by the Russian Foundation for Basic Research, project 14-04-01423a and DS by SIU foundation (NCM-RU/10066) CIMO-FIRST and Academy of Finland (project \# 257748).

\section{REFERENCES}

Berglund, H., Hottola, J., Penttilä, R. \& Siitonen, J. 2011. Linking substrate and habitat requirements of wood-inhabiting fungi to their regional extinction vulnerability. Ecography 34(5): 864-875. http://dx.doi.org/10.1111/j.16000587.2010.06141.x

Bernicchia, A. \& Gorjón, S. P. 2010. Corticiaceae s. l. Fungi Europaei, vol. 12. Ed. Candusso. 1009 pp.

Bondartseva, M. A. 1986. Wood-decaying fungi of the Central Forest Reserve (in Russian). Novitates systematicae plantarum non vascularium 23: 103-110.

Bukharova, N. V. \& Zmitrovich, I. V. 2014. Aphyllophoroid fungi of the "Bastak" reserve (in Russian, English summary). Mikologiya i Fitopatologiya 48(6) 343-354

Chastukhin, V. Y. 1948. Destruction of plant debris in spruce forests. I Macroscopic fungi (in Russian). Biologija pochv 2: 13-91.

Chastukhin, V. Y. \& Nikolaevskaya, M. A. 1969. Biological distruction and resynthesize of organic compounds in nature (in Russian). Leningrad. 325 pp.

Eriksson, J. \& Ryvarden L. 1973. The Corticiaceae of north Europe, vol 2. Oslo. Pp. 112-121.

Karpov, V. G. 1983. Regulation factors of spruce forest ecosystems (in Russian). Leningrad. 317 pp.

Kotiranta, H. \& Saarenoksa, R. 2005. The genus $\mathrm{Ba}-$ sidiodendron (Heterobasidiomycetes, Tremellales) in Finland. Annales Botanici Fennici 42: 11-22.

Kotiranta, H., Saarenoksa, R. \& Kytövuori, I. 2009. Aphyllophoroid fungi of Finland. A check-list with ecology, distribution, and threat categories. Norrlinia 19: 1-223.

Kotiranta, H., Shiryaev, A.G. \& Spirin, V. 2016. Aphyllophoroid fungi (Basidiomycota) of Tuva Republic, southern Siberia, Russia. Folia Cryptogamica Estonica 53: 51-64.

Kotkova, V. M. 2012a. Additions to the biota of aphyllophoroid fungi (Basidiomycota) of Central-forest reserve (Tver Region) (in Russian). Vestnik TvGU. Seriya "Biologiya i ekologiya". 28(25): 119-123.

Kotkova, V. M. 2012b. New data on aphyllophoroid fungi of Central-forest state biosphere reserve (in Russian). Mikologiya i Fitopatologiya. 46(6): 361-364.

Kotkova, V. M. 2012c. The study of aphyllophoroid fungi in the forests of Central-forest state biosphere reserve (in Russian). Dinamika mnogoletnih processov $v$ jekosistemah Central'no-Lesnogo zapovednika. Tr. CLGPBZ. 6: 307-318.

Kotkova, V. M. 2014a. Additions to the biota of aphyllophoroid fungi (Basidiomycota) of Central-forest reserve (Tver Region) II (in Russian). Vestnik TvGU. Seriya "Biologiya i ekologiya". 28(25): 119-123.

Kotkova, V. M. 2014b. Fungi of the Central-forest reserve (Annotated species list) (in Russian). Flora i fauna zapovednikov. 122: 1-94.

MycoBank (http:/ / www.mycobank.org/), 31.10.2016. 
Pugachevsky, A. V. \& Shaposnikhov, E. S. 1999. Age dynamics of trees and the development of forest communities (in Russian, English summary). In: Smirnova, O. V. \& Shaposnikhov, E. S. (eds.). Forest successions in protected areas of Russia and problems of biodiversity conservation. StPetersburg. 325-333.

Raitviir, A. G. 1967. Keys to the Heterobasidiomycetes of the USSR (In Russian). Leningrad. $113 \mathrm{pp}$.

Renvall, P. 1995. Community structure and dynamics of wood-rotting Basidiomycetes on decomposing conifer trunks in northern Finland. Karstenia 35: $1-51$.

Roberts, P. 1994. Globose and ellipsoid-spored Tulasnella species from Devon and Surrey, with a key to the genus in Europe. Mycological Research 98: 1431-1452. http://dx.doi.org/10.1016/S09537562(09)81075-7

Roberts, P. \& Hauerslev, K. 1997. Tulasnellales Rea. In: Hansen, L. \& Knudsen, H. (eds). Nordic Macromycetes 3. Heterobasidioid, Aphyllophoroid, and Gastromycetoid Basidiomycetes. Copenhagen. Pp. 115-118.

Ryvarden, L. \& Melo, I. 2014. Poroid fungi of Europé. Synopsis Fungorum 31: 1-455.

Sell, I. \& Kotiranta, H. 2011. Diversity and distribution of aphyllophoroid fungi growing on Common Juniper (Juniperus communis L.) in Estonia. Folia Cryptogamica Estonica 48: 73-84.

Shiryaev, A. G., Kotiranta, H., Mukhin, V. A., Stavishenko, I. V. \& Ushakova, N. V. 2010. Aphyllophoroid fungi of Sverdlousk region, Russia: biodiversity, distribution, ecology and the IUCN threat categories. Yekaterinburg. 303 pp.
Sorokin, A. S. 2002. The Red Book of the Tver region (In Russian). Tver. 256 pp.

Storozhenko, V. G. 2007. Results and prospects of phytopathological research in forests of the Central Forest State Biosphere Reserve (In Russian). Kompleksnye issledovanija $v$ Central'no-Lesnom gosudarstvennom prirodnom biosfernom zapovednike: ih proshloe, nastojashhee $i$ budushhee. Tr. CLGPBZ. 4: 43-52.

Tomsovský, M. \& Jankovský, L. 2008. Validation and typification of Laetiporus montanus. Mycotaxon. 106: 289-295.

Trofimov, T. T. 1948. The main achievements of the reserves in the study of flora and vegetation in RSFSR (In Russian). Nauchno-metodicheskie zapiski. Glavnoe upravlenie po zapovednikam. 10: 9-52.

Trutnev, Y. P. 2008. Red Data Book of the Russian Federation (Plant and Fungi) (In Russian). Moscow. 855 pp.

Viner I. A. 2012. Some features of the development of wood-decaying fungal community on spruce from windfalls of different age (In Russian). Mnogoletnie processy $v$ prirodnyh kompleksah zapovednikov Rossii. Materialy Vserossijskih Nauchnyh Konferencij 119-124.

Viner I. A. 2015. Polyporoid and corticioid Basidiomycetes in pristine forests of the Pechora-Ilych Nature Reserve, Komi Republic, Russia. Folia Cryptogamica Estonica 52: 81-88. http: / / dx.doi. org/ 10.12697/fce.2015.52.10 
92 Folia Cryptog. Estonica 